\title{
Current Status and Future Directions in Colorectal Cancer
}

\author{
B. Meyer $^{1} \cdot$ Chandrakanth Are $^{1}$
}

Published online: 23 October 2017

(C) Indian Association of Surgical Oncology 2017

Colorectal cancer is the fourth most common cancer in the world with 1.3 million new cases each year and a 5 -year prevalence rate of 3.2 million $[1,2]$. There were an estimated 693,333 deaths due to colorectal cancer (CRC) in 2012. When looking at India and the USA, the incidence, mortality, and prevalence rates are all consistently higher in the USA although the incidence is higher in males in both countries [3]. In the USA, CRC falls behind prostate, breast, and lung as the fourth most common cancer. In India, it is the fifth most common cancer following breast, cervix/uteri, lip/oral cavity, and lung cancer [3].

North America, Australia, and Europe have higher rates of CRC when compared to other parts of the world. It is thought that "the westernized lifestyle" of physical inactivity, poor diet, obesity, and increased alcohol consumption and long-term smoking attributes to this higher burden $[4,5]$. In contrast, registries in Asia, Africa, and South America have some of the lowest rates of CRC [4]. A review of the 12 population-based cancer registries from India (Benaguluru, Barshi, Bhopal, Chennai, Delhi, Mumbai, Ahmedabad, Karunagappally, Kolkata, Nagpur, Pune, and Thiruvananthapuram) again demonstrated that the incidence of colon and rectal cancer is significantly lower in India for both genders when compared to that of the West. In these registries, colon and rectal cancer is ranked tenth among all cancers. It was thought that until recently, there were fewer known risk factors for colorectal cancer in India. The typical diet in India is lower in calories and consists of more fruits

Chandrakanth Are

care@unmc.edu

1 Division of Surgical Oncology, Department of Surgery, Fred and Pamela Buffett Cancer Center, University of Nebraska Medical Center, Omaha, NE 68198, USA and vegetables. This combined with a higher level of physical activity translates to a lower obesity rates $[6,7]$. Unfortunately, India's low incidence rate is also associated with a low 5-year survival rate [7].

Even though the rates of CRC are much higher in the USA, the incidence and mortality rates have been decreasing in the past 20 years. According to the American Cancer Society, this is likely due to CRC screening tests that allow for early detection and removal of polyps before progression to cancer. The incidence rates declined by $3.7 \%$ per year among those 50 and older from 2006 to 2010; conversely, the incidence rate increased by $1.8 \%$ per year among those younger than 50 . Mortality rates have also declined, from 2005 to 2014, by $2.5 \%$ per year [5].

Patients with CRC usually present with rectal bleeding, pain, or a change in bowel habits. Occasionally, patients will present with a malignant large bowel obstruction. The article by Patil et al. demonstrated that patients in India presented with the same symptoms as in the USA; however, the patients were typically younger and presented at a later stage. In the article by Ashish et al., CRC data in the young $(<45)$ was compared with that of the old $(>45)$ and those $<45$ were found to have poor histology, nodepositive disease, and more cases of rectal cancer. Nonetheless, there was no difference in overall survival at 3 years in these two groups in India. In the article by Muralee et al., the outcomes of patients older than 70 with $\mathrm{CRC}$ and other comorbidities were investigated. The article showed curative treatment is feasible and with good outcomes in this patient population.

Once a diagnosis of CRC is made, the stage must be determined. This is achieved with the help of a CT of the chest, abdomen, and pelvis and an MRI of the pelvis or endoscopic ultrasound if staging rectal cancer. In addition, labs such as CEA and biomarkers of the tumor are obtained. Koval et al. 
found that higher CEA levels were associated with higher TNM stage, tumor size, and microsatellite instability (MSI) status. Similarly, C-reactive protein-albumin ratio can assist in predicting prognosis in those patients with unresectable CRC as shown by Masahide et al. Yuji et al. looked at the survival rates in conjunction with the tumor biomarkers RAS, BRAF, and MSI. Their article showed that metastatic CRC survival rates have increased over the past 20 years due to patient selection for treatment plans based on these biomarkers.

Typically, patients with CRC will undergo different combinations of surgery, chemotherapy, and radiation. The surgical procedure chosen depends on the location of the tumor and patient factors and may include partial colon resection, low anterior resection, or an abdomino-perineal resection. While performing a distal colon or rectal resection, placing a diverting loop ileostomy is occasionally done with the plan to take it down in 6-8 weeks. In the article by Madhu et al., they looked at using the triple test or in order to predict anastomotic integrity. The triple test consists of the classical air leak test, inspecting the donuts from the stapler for completeness and a DRE to assess the anastomosis. In this prospective study, the leak rate was $7 \%$. This showed that the triple test was a reliable predictor of the anastomosis integrity and if negative, a diverting stoma can be avoided, even with those who received radiation preoperatively.

Minimally invasive surgery is becoming increasingly popular in the treatment of CRC. Nam et al. looked at using a minimally invasive approach for both colon and rectal cancers. Typically, a minimally invasive approach allows for faster recovery of bowel function, reduced postoperative pain, and shorter hospitalizations. Laparoscopic colon resections are widely done for colon cancer; however, for rectal cancer, the operation is technically more difficult due to dissection in the deep and narrow pelvis. Robotic surgery is becoming more popular for rectal cancer as it allows for better visualization in the pelvis and improved ergonomics and dexterity when compared to laparoscopic surgery for total mesorectal excision.

Chemotherapy has evolved in the past 20 years and now, there are multiple combination regimens available. The most popular include FOLFOX (leucovorin, 5-FU, and oxaliplatin), FOLFIRI (leucovorin, 5-FU, and irinotecan), and in some cases, a drug that targets VEGF or EGFR, bevacizumab (Avastin) or cetuximab (Erbitux), respectively. Different combinations of chemotherapy and radiation are given to patients based on stage, tumor characteristics, and presence of biomarkers.

Due to advances in the treatment of CRC and early detection, the survival rates have increased in the USA, with a 5year survival of $65 \%$ at all stages [3]. The USA also has colorectal screening programs widely available. These include colonoscopy, fecal occult blood testing, CT colonography, flexible sigmoidoscopy, double contrast barium enema, or
DNA stool testing. When evaluating the CRC burden worldwide, it is important to consider whether screening programs exist as they can transiently increase the incidence of CRC [4]. These numbers will then decrease as more precancerous polyps are detected early before turning into a cancer. This is thought to be a reason for the recent decline in $\mathrm{CRC}$ rates in the USA.

CRC detection and treatment has certainly made strides in the past few decades, especially in the USA and other economically developed countries [1]. In other parts of the world such as India, the incidence still remains low and is less than half of that in the USA. Nonetheless, the rate of CRC in India and other emerging economies such as Eastern Europe and South America is rising. In India, it is predicted to rise approximately by $80 \%$ in 2035 , with an incidence of 114,986 new cases and a mortality of 87,502 [1]. Efforts should be made for increasing formal screening programs in the age-appropriate population. Identifying people at higher risk because of family history, history of inflammatory bowel disease, or genetic conditions and targeting them for screening should be encouraged. Finally, educating individuals on risk factors and what should prompt them to seek care combined with a multidisciplinary approach will help tackle the rising CRC burden in India and improve outcomes.

\section{References}

1. Ferlay J, Dikshit R, Elser S, Mathers C, Rebelo M, Parkin DM, Forman D, Bray F (2013) GLOBOCAN 2012 v1.0, Cancer incidence and mortality worldwide: IARC CancerBase No. 11 [internet]. Lyon: International Agency for Research on Cancer. Available from: http://globocan.iarc.fr/Pages/burden_sel.aspx. Accessed 25 Aug 2014

2. Bray F, Ren JS, Masuyer E, Ferlay J (2013) Estimates of global cancer prevalence for 27 sites in the adult population in 2008 . Int $\mathrm{J}$ Cancer 132(5):1133-1145. https://doi.org/10.1002/ijc.27711 Available from: http://globocan.iarc.fr/Default.aspx. Accessed 21 Aug 2014

3. Ferlay J, Dikshit R, Elser S, Mathers C, Rebelo M, Parkin DM, Forman D, Bray F (2013) GLOBOCAN 2012 v1.0, Cancer incidence and mortality worldwide: IARC CancerBase No. 11 [Internet]. Lyon: International Agency for Research on Cancer. Available from: http://globocan.iarc.fr/Pages/fact_sheets population.aspx Accessed 07 August 2017

4. Center MM, Jemal A, Smith RA, Ward E (2009) Worldwide variations in colorectal cancer. CA Cancer J Clin 59:366-378. https://doi. org/10.3322/caac. 20038

5. American Cancer Society (2014) Cancer facts and figures 2014. http://www.cancer.org/research/cancerfactsstatistics/cancer factsfigures2014/. Accessed 06 Aug 2017

6. National Cancer Registry Programme (2010) Development of an atlas of cancer in India. First all India report: 2001-2002. http:// www.canceratlasindia.org/chapter3 5.aspx. Accessed 21 Aug 2014

7. Pathy S, Lambert R et al (2012) The incidence and survival rates of colorectal cancer in Indian remain low compared with rising rates in East Asia. Dis Colon Rectum 5(8):900-906 